Vivre, penser, écrire en exil

\title{
Les écrivains russes blancs en France. Un entre- deux identitaire (1919-1939)
}

White Russian Writers in France. A Half-Way Identity (1919-1939)

Escritores rusos blancos en Francia. Una identidad intermedia

\section{Ralph Schor}

\section{(2) OpenEdition}

Journals

Édition électronique

URL : https://journals.openedition.org/remi/8553

DOI : 10.4000/remi.8553

ISSN : $1777-5418$

Éditeur

Université de Poitiers

Édition imprimée

Date de publication : 1 mars 2017

Pagination : 11-26

ISBN : 979-10-90426-30-6

ISSN : 0765-0752

\section{Référence électronique}

Ralph Schor, "Les écrivains russes blancs en France. Un entre-deux identitaire (1919-1939) », Revue européenne des migrations internationales [En ligne], vol. 33 - n¹ | 2017, mis en ligne le 01 mars 2019, consulté le 15 avril 2022. URL : http://journals.openedition.org/remi/8553 ; DOI : https://doi.org/ 10.4000/remi.8553 


\section{Les écrivains russes blancs en France. Un entre-deux identitaire (1919-1939)}

\section{Ralph Schor ${ }^{1}$}

La révolution bolchevique de 1917 lança de nombreux Russes sur les chemins de l'exil. En France, leurs effectifs atteignirent 32247 personnes en 1921 et 67219 en 1926. Par la suite, les chiffres se stabilisèrent entre 70000 et 80000 individus (Struve, 1996). Parmi les réfugiés figuraient de nombreux écrivains de premier plan, dont Ivan Bounine, premier Russe ayant obtenu, en 1933, le prix Nobel de littérature, Marc Aldanov, Nina Berberova, Ivan Chmeliov, Gaïto Gazdanov, Romain Gary, Nina Goufinkel, Zenaïde Hippius, Georges Ivanov, Vladislav Khodassevitch, Alexandre Kouprine, Dimitri Merejkovski, Vladimir Nabokov, Irène Nemirovski, Zoé Oldenbourg, Alexis Remizov, Henri Troyat, Tarassov de son vrai nom, Marina Tsvetaeva, Boris Zaïtsev, etc. Paris devint alors la capitale des lettres russes en exil ; en comparaison, Prague, Belgrade, Varsovie, Riga où s'étaient installés d'autres écrivains faisaient figure de foyers provinciaux (Etkind et al., 1987-1990 ; Waegemans, 2003). Nabokov, revenant plus tard sur cette période, dira :

"À de rares exceptions près, toutes les forces créatrices d'esprit libéral - poètes, romanciers, critiques, historiens, philosophes et autres - avaient quitté la Russie de Lénine et de Staline [...]. Les livres produits in vacuo par des écrivains émigrés semblent aujourd'hui, quels que soient les défauts propres à chacun d'entre eux, plus durables et plus dignes d'être lus que ces monologues politiques intérieurs, serviles, curieusement provinciaux et conventionnels qui coulèrent durant ces mêmes années des plumes de jeunes auteurs soviétiques qu'un État paternel fournissait en encre " (Nabokov, 1961).

\section{Les conditions de l'exil}

L'arrivée et l'installation des réfugiés russes étaient largement conditionnées par des facteurs, les uns positifs, les autres négatifs.

La mode russe qui se développa au cours des années 1920 donna des nouveaux venus une image très favorable et engendra même un véritable engouement. En effet, les Français voyaient leurs hôtes comme les victimes romanesques d'un spectaculaire retournement de situation, des élus de la fortune tombés dans une sombre misère. Les chauffeurs de taxi originaires de

1 Historien, Professeur émérite, Université de Nice-Sophia-Antipolis, 98 boulevard Edouard Herriot, BP 369, 06007 Nice cedex ; e.schor@ wanadoo.fr 
l'empire des tsars, certes nombreux à Paris, étaient tous regardés comme des princes ou des généraux désormais privés de leurs titres et de leur fortune. Les émigrés éveillaient aussi l'intérêt par le portrait psychologique stéréotypé qui en était brossé : selon l'opinion commune, ils incarnaient les fascinants mystères de l'âme slave, tour à tour exaltés et abattus, courtois et sauvages, ascètes et jouisseurs, fatalistes et entreprenants, Européens raffinés et Asiatiques inquiétants. Leur musique sensuelle était réputée refléter ces contradictions. Plus prosaïquement, certains Français voulaient recueillir le témoignage des exilés et le récit de leurs expériences pour comprendre la révolution de 1917 et en limiter les effets en occident.

La mode russe aux motivations diverses s'incarna sous de multiples formes. Les artistes jouissaient d'une immense notoriété. Igor Stravinsky était déjà connu du public avant la guerre, surtout pour la retentissante création de son ballet $L e$ Sacre du printemps au théâtre des Champs Élysée le 29 mai 1913 ; revenu en France où, disait-il, " battait alors le pouls de l'activité mondiale " (Stravinsky, 1935), il continua à collaborer avec Serge de Diaghilev, flamboyant directeur des Ballets russes, et connut le succès. Toujours dans le domaine de la danse, Serge Lifar, fêté par le Tout-Paris, fut nommé maître de ballet de I'Opéra en 1930. Les acteurs de théâtre Ludmilla et Georges Pitoëff produisaient une forte impression avec leurs créations des œuvres de Tchekov, Pirandello, Anouilh. Sur les écrans du cinéma muet, Ivan Mosjoukine était considéré comme une étoile de première grandeur. De nombreux magasins de mode ou de produits alimentaires, des expositions d'art, des salons de thé, des restaurants, des cabarets très courus et peints avec brio par Joseph Kessel dans son roman Nuits de princes (1927), popularisaient tout ce qui venait de l'empire disparu, comme le relevait le romancier Gaïto Gazdanov :

« II existait à Paris des dizaines de magasins et des restaurants russes. On y vendait des produits du pays; dans les restaurants on servait des plats russes : des blinis, des feuilles de chou farci et l'inévitable bortsch " (Gazdanov, 1991).

La mémorialiste Nina Gourfinkel confirmait :

"Le grand public découvrait non sans ébahissement ce qui, jusque-là, n'était connu que de l'élite. Avec la multiplication d'expositions, de spectacles, de restaurants et de cabarets russes, le Paris qui s'amuse trouvait un nouveau style de boîte de nuit où, sous la frivolité, semblait frissonner une âme pleine de passion " (Gourfinkel, 1953).

Les Russes, stimulant la curiosité, inspirèrent des enquêtes journalistiques et des traductions de leurs œuvres. Des écrivains français parmi les plus connus, comme Pierre Benoit (Cavalier 6, 1933), Francis Carco (Verotcka l'étrangère ou le goût du malheur, 1923), Maurice Bedel, romancier à succès et lauréat du prix Goncourt (Molinoff Indre-et-Loire, 1928), s'emparèrent des exilés et en firent des héros de romans, de nouvelles, de pièces de théâtre : dans l'entre-deux-guerres, un quart des personnages étrangers figurant dans les œuvres littéraires françaises étaient d'origine russe (Schor, 1985).

Autre facteur favorable à leur insertion sociale, les Russes se trouvèrent intégrés dans de nombreuses associations humanitaires et des réseaux de communication intellectuelle. Ainsi le Comité de secours aux écrivains et savants russes en France dispensait une aide matérielle et organisait des 
événements culturels ouverts aux Français, lectures, colloques, concerts, représentations théâtrales. Les Amis des lettres, dissidents de la précédente organisation, fonctionnaient sur le même modèle, tout comme l'Union des jeunes poètes et écrivains et le Cercle Kotchevie ou Camp des nomades. Les écrivains se retrouvaient fréquemment dans les cafés, telles la Rotonde et la Coupole à Montparnasse. La sociabilité de l'exil était encore entretenue par les salons littéraires. Le couple Dimitri Merejkovski et Zénaïde Hippius organisait à son domicile des soirées culturelles très courues, dites la Lampe verte, du nom d'un cercle jadis fréquenté par Pouchkine à Saint-Pétersbourg. Nina Berberova se rendait chez le riche Maxime Vinaver, ancien député à la douma et rédacteur aux Dernières Nouvelles:

« Dans les années 1925-1926 se tenait chez les Vinaver un des salons littéraires russes de Paris. Leur immense appartement, situé dans les beaux quartiers, avec ses tapisseries, ses candélabres, son piano à queue, sa bibliothèque garnie de livres rappelait les appartements pétersbourgeois d'autrefois. Une trentaine de personnages assistaient aux réunions. Il y avait les célébrités comme Maklakov, Milioukov, le couple Merejkovski et Bounine, mais aussi les jeunes talents prometteurs qui fréquentaient les cafés de

Montmartre et écrivaient dans l'hebdomadaire Le Chaînon, dont l'éditeur et le rédacteur était Vinaver " (Berberova, 1992).

Les intellectuels se retrouvaient encore lors de nombreux banquets et fêtes commémoratives comme la Fête de l'université, célébrée avec la Sainte-Tatiana le 25 janvier et le Jour de la culture russe qui coïncidait avec la naissance de Pouchkine le 6 juin. Le 125e anniversaire de la naissance de celui-ci en 1924 et le centenaire de sa mort en 1937 donnèrent lieu à des manifestations importantes.

Une presse importante, forte de 167 titres, constituait un lien entre les émigrés dispersés, un élément de cohésion sociale et intellectuelle. Parmi les quotidiens figuraient les Dernières Nouvelles qui tiraient environ à 35000 exemplaires et la Renaissance dont la rubrique littéraire était tenue par le poète Khodassevitch. La Russie et le monde moderne paraissaient chaque semaine. Parmi les revues culturelles se détachaient les Nombres (Tchiska), où écrivaient les jeunes épris de modernité, et les classiques Annales contemporaines dont Nina Berberova disait :

"La collaboration aux Annales contemporaines représentait dans l'émigration une sorte de distinction honorifique. Avec ses soixante-dix tomes, cette revue est un véritable monument de la littérature. Ce n'était pas une revue d'avant-garde, mais, durant un quart de siècle, y parurent des œuvres importantes " (Berberova, 1992).

En matière d'enseignement les Russes manifestèrent leur volonté de former les jeunes pour leur transmettre le patrimoine culturel de la vieille patrie et préparer les futurs cadres du pays libéré du pouvoir bolchevik. À cet effet furent créés un lycée russe en 1921, une Université populaire russe en 1929, des instituts supérieurs en sciences sociales, commerciales et naturelles, une Académie de philosophie religieuse, l'institut de théologie Saint-Serge en 1926.

Enfin, dernière condition favorable, les Russes, intellectuels reconnus et souvent francophones, entretinrent des relations suivies avec leurs homologues français. Dans leurs souvenirs, ils énumèrent le nom des nombreux écrivains qu'ils rencontrèrent au fil du temps, comme Maurice Barrès, Paul Valéry, André 
Gide, André Malraux, Jean Cocteau, Jacques Maritain, Charles Vildrac, Jean Giraudoux, Sacha Guitry, Anna de Noailles, Henri de Montherlant, Marcel Aymé, André Maurois, Gabriel Marcel, ainsi que des hommes politiques, surtout Édouard Herriot, Louis Barthou, Paul Painlevé. Ces contacts leur permirent d'obtenir des recommandations pour accéder à des journaux et à des éditeurs français.

Cependant, I'accueil trouvait ses limites et les émigrés rencontrèrent des difficultés sérieuses. En premier lieu, la mode russe, éphémère par nature, déclina dès la fin des années 1920. L'intérêt passager n'était pas forcément synonyme de sympathie pour les Russes, mais plutôt de curiosité qui, une fois satisfaite, laissa place à une certaine indifférence. En outre, dans les années 1930, la France fut touchée par la grande crise économique née aux États-Unis en 1929, de sorte que les préoccupations matérielles devinrent prioritaires. La population appauvrie se détourna des produits russes au prix onéreux et particulièrement des cabarets, ainsi que l'observa le journaliste Pierre de Régnier dans Gringoire, le 6 janvier 1933 :

"Ce ne fut pas forcément le genre russe qui cessa de plaire, mais bien normalement le genre cher, car ces endroits étaient de beaucoup les plus ruineux, les plus snobs et les plus aristocratiques au sens intégral du mot ".

Tandis que le public se détournait des Russes blancs, I'intérêt pour I'URSS grandissait. La famine qui régna au pays des soviets en 1921 éveilla la compassion des Français qui envoyèrent des secours prélevés en partie sur ceux qui auraient pu bénéficier aux émigrés. Puis la France reconnut, de jure, le régime soviétique en 1924. Les intellectuels français épris de modernité manifestèrent de la curiosité pour un jeune pays où semblait naître un art d'avant-garde détaché des conventions anciennes. Zoé Oldenbourg qui avait émigré avec ses parents analyse finement cet état $d^{\prime}$ esprit :

" Des vaincus, nous l'étions, et condamnés à garder la tête basse. Notre témoignage était d'avance discrédité - une bonne partie de l'intelligentsia française, et la plus saine peut-être, affichait à l'égard de la révolution d'0ctobre et du régime soviétique un enthousiasme naïf, fait surtout d'ignorance ; et nous qui en savions plus long qu'eux étions considérés comme des menteurs, des réactionnaires, des "gardes blancs", voire des traîtres " (Oldenbourg, 1977).

Dans le même temps, les Russes réfugiés en France se divisaient selon plusieurs lignes de fracture. La première de celles-ci courait de part et d'autre de la date de départ. La deuxième vague d'émigrés, souvent composée de socialistes qui avaient soutenu la révolution à ses débuts, méprisaient leurs compatriotes partis dès les premiers troubles, considérés comme des monarchistes réactionnaires, des individualistes, voire des lâches, ayant fui les souffrances et la famine endurées par ceux qui étaient d'abord restés pour mener à bien une expérience politique et sociale nouvelle. Un autre type de division séparait les générations. Les plus âgés, comme Bounine, Merejkovski, Kouprine, Zaïtsev, déjà connus en Russie avant la révolution, bénéficiaient d'un statut social supérieur et parvenaient plus facilement à publier leurs œuvres ; en revanche, les jeunes, comme Nina Berberova, Poplavski et même Nabokov, connaissaient une vie matérielle plus précaire et accédaient difficilement aux structures de 
publication. Le choc des personnalités entretenait de multiples mésententes. Ainsi la poétesse MarinaTsvetaeva, entière et tourmentée, affichant brutalement ses choix, était rejetée à la fois par les progressistes, car elle se disait fidèle à la vieille Russie et les conservateurs, car elle proclamait son admiration pour le poète soviétique Maïakovski. De Bounine, prestigieux lauréat du prix Nobel, elle disait durement : "Je n'ai encore jamais vu Bounine. Je ne l'aime pas : un barine froid, cruel, imbu de lui-même [...]. Gorki c'est une époque. Tandis que Bounine c'est la fin d'une époque " (Tsvetaeva, 2003). Enfin les choix politiques effectués dans l'exil constituaient un gisement infini de divisions. Zoé Oldenbourg constatait : "Là où il y a deux Russes, il y a trois opinions différentes " (Oldenbourg, 1977). Nicolas Struve ajoute : " ils se détestaient tellement les uns les autres qu'il était difficile de réunir vingt personnes sans que dix d'entre elles ne soient les ennemies des dix autres. Et si elles ne l'étaient pas, elles le devenaient surle-champ " (Struve, 1996). Le point central était l'attitude à adopter à l'égard de I'URSS. L'extrême droite monarchiste rêvait de restaurer le tsarisme d'avant 1917, comme si rien ne s'était passé. Les libéraux comme Nabokov souhaitaient une monarchie constitutionnelle ou une République modérée et démocratique. Certains, tout en restant en France, étaient, selon les termes de Dimitri Sesemann, "convertis à la soviétophilie ", mais acceptaient " une servitude volontaire [...] en dépit des cris et des gémissements qui parvenaient, quoiqu'assourdis, des bagnes de Kolyma et de Sibérie "(Sesemann, 2001). Certains, saisis par le mal du pays, tels Alexis Tolstoï, Nicolas Klepinine, Serge Efron, mari de Tsvetaeva, finirent par revenir en URSS. Marina Tsvetaeva hésita deux ans avant de rejoindre son époux ; à peine fut-elle arrivée que celui-ci fut fusillé ; elle-même, exilée loin des siens, se suicida. En vérité, ceux qui réfléchissaient étaient marqués par la sinistre figure de Staline. Nina Berberova, tentée par le retour, ne donna pas suite à cette velléité, car le " tsar rouge " lui inspirait " de I'indignation, du dégoût, de l'humiliation et de la terreur " (Berberova, 1992).

La plupart des Russes se plaignaient de l'isolement dans lequel ils vivaient et incriminaient la distance prise à leur égard par leurs hôtes. "Le Français n'a à faire que de lui-même ", notait aigrement Marina Tsvetaeva dans une lettre de 1932 (Tsvetaeva, 2003). Zoé Oldenbourg ajoutait : " J'ai fait toutes mes études en France, de dix à dix-huit ans, dans une école libre puis sans un lycée, jamais aucune de mes camarades ne m'invita chez elle "(Oldenbourg, 1977). II est juste d'observer que ce sentiment de solitude pouvait aussi être imputé aux exilés. En effet, beaucoup d'entre eux pointaient les différences de mentalité et de comportement qu'ils voyaient chez les Français et, avec une certaine dose d'intolérance, refusaient d'accepter ces dissemblances. Sans doute étaient-ils, selon les cas, trop âgés, trop attachés à leur pays, trop peu familiers de la langue de Molière, trop accablés par les épreuves pour faire mouvement en direction de leurs hôtes. Tsvetaeva confiait dans une lettre de 1927 : "Je ne change pas au contact des gens [...]. À vrai dire, je suis tellement malmenée par la vie que je ne ressens rien " (Tsvetaeva, 2003). Aussi beaucoup d'émigrés se repliaient-ils sur un cercle étroit de compatriotes.

La gêne matérielle pesait lourdement sur la vie quotidienne des exilés. Certains avaient conservé une partie de leur fortune, comme les parents d'Irène Nemirovski ou ceux d'Henri Troyat. Mais beaucoup, imprévoyants, s'appauvrissaient rapidement comme ces héros d'une nouvelle d'Ivan Bounine : " II y eut de moins en moins d'argent, nous dépensâmes nos derniers sous pour aller à 
Monte-Carlo où nous perdîmes tout "(Bounine, 2003). La plupart, partis rapidement de Russie, sans avoir prévu les moyens de subsister en exil, vivaient dans la précarité. Le statut de réfugié Nansen permettait d'obtenir des papiers, mais non d'occuper un emploi, en vérité difficile à trouver en temps de crise, ce qui faisait dire à Nina Gourfinkel : "Le droit de séjour était accordé au réfugié, mais non le droit au travail. Autrement dit : on voulait bien le sauver, mais non lui permettre de vivre " (Gourfinkel, 1953). Les petits métiers furent généralement la règle. Nobokov devint professeur d'anglais et de tennis, Nina Berberova brodeuse, enfileuse de perles, figurante de cinéma. André Trofimoff observe que nombre de ses compatriotes qui auraient pu rester "fonctionnaires falots " ou " hommes du monde désœuvrés" se découvrirent en exil des " talents insoupçonnés ": " de nombreux Russes se révélèrent adroits commis-voyageurs, cuisiniers, joueurs de balalaïka "; lui-même, ancien conservateur des musées impériaux, se mua en brocanteur (Trofimoff, 1937). Autres ressources : solliciter des aides auprès de divers comités de secours ou d'admirateurs, comme Nabokov qui recevait des mandats de Rachmaninov qu'il n'avait cependant jamais rencontré ; emprunter ainsi que le faisait Alexis Tolstoï à qui Bounine prêtait ces propos : " J'ai tapé tous ceux que j'ai pu et j'ai trente-sept mille francs de dettes... Mille francs jusqu'à vendredi, sinon je me fais sauter la cervelle " (Bounine, 1950). Les écrivains pouvaient aussi placer des articles, des traductions, des recensions, etc., mais les droits d'auteur étaient chichement mesurés. II en résultait une insécurité matérielle inquiétante, une "vie de bohême " comme disaient eux-mêmes les exilés : "Nous avions été des personnages considérables et considérés. À Paris nous n'étions rien ", note Zoé Oldenbourg (Oldenbourg, 1977). Les réfugiés s'installaient dans des hôtels modestes, situés dans les quartiers populaires. Nina Krivochéine se rappelle : "Notre logis était infect, sale, la maison grouillait de punaises " (Krivochéine, 1987). La chambre meublée où vivait Nobokov était si exigüe que, pour laisser dormir sa femme et son fils, il se réfugiait dans le cabinet de toilette ; là, il utilisait comme bureau une valise posée sur le bidet devant lequel il se mettait à genoux. Tsvetaeva vivait avec son mari et ses deux enfants dans une pièce unique, munie d'une seule table sur laquelle elle pouvait seulement écrire le soir, les tâches ménagères accomplies et la table débarrassée. Elle était jugée distante, car elle refusait nombre d'invitations; en fait elle ne possédait pas la robe noire et les chaussures vernies alors d'usage en société. Tous mesuraient leurs achats de produits alimentaires et, pour se réchauffer l'hiver, passaient de longues heures dans les cafés.

Quel que fût le cadre, positif ou négatif, dans lequel s'exerçait la création littéraire, les écrivains se partageaient en deux groupes en fonction de leur génération.

\section{La vieille génération : la douleur de l'exil}

La plupart des écrivains russes installés en France se considéraient comme les plus authentiques gardiens de l'héritage culturel national et refusaient tout dialogue avec leurs confrères restés en URSS. Cet état d'esprit se révélait particulièrement vivace chez les aînés. Pour autant, ceux-ci et les critiques littéraires se demandaient s'il était possible de demeurer un authentique écrivain russe hors de Russie. En effet, remarquaient-ils tristement, leur patrie, telle qu'ils 
I'avaient connue, n'existait plus ; ils se trouvaient coupés de leurs sources d'inspiration, de la tradition nationale, de leur public naturel. S'ils continuaient à écrire, resteraient-ils en accord avec leur art tel qu'ils I'avaient pratiqué avant la révolution et ne risquaient-ils pas de trahir l'essence de la terre natale ? S'ils se résignaient à changer, même imperceptiblement, et a fortiori s'ils s'intégraient à la France, même superficiellement, pouvaient-ils encore se prévaloir du titre, si cher à leur cœur, d'écrivain russe ? Aussi beaucoup se montraient-ils pessimistes sur l'avenir d'une littérature d'exil. À cette question, quelques-uns répondaient que l'identité d'un auteur ne dépendait pas du lieu de son séjour, mais de son paysage intérieur façonné par l'expérience passée et entretenu par le souvenir. Telle était la position de Banine :

"La patrie n'est pas déterminée par le territoire, elle est l'immutabilité de la mémoire et du sang. Cesser d'être Russe, oublier la Russie, seul peut le craindre celui qui la pense comme extérieure à lui-même. Celui qui la porte en lui ne la perdra qu'avec la vie "

(Banine, 1968).

Quoi qu'il en fût de la réponse, les écrivains en exil continuèrent à écrire d'abondance et convoquèrent sous leur plume toutes les images du passé (Schor, 2013). En vérité cette orientation les menait directement à la nostalgie du temps jadis, quitte à se couper de la réalité qui leur était contemporaine. Un héros de Gazdanov soupirait : "Le monde dans lequel nous avons vécu n'existe plus que dans notre imagination " et il ajoutait, en généralisant : "Les meilleurs devenaient des rêveurs évitant tout contact avec une réalité dérangeante " (Gazdanov, 1991). "La Russie, c'était un rêve ", assurait Remizov (Berberova, 1992), "une idéalisation forcenée du passé ", ajoutait Nina Gourfinkel (Gourfinkel, 1953). Les images de ce rêve éveillé véhiculaient de nombreux souvenirs personnels, la description de grandes plaines parsemées d'étangs et de saules, l'évocation des nuits blanches de Saint-Pétersbourg et des musiques tziganes, " un passé moisi, rance, baigné de vapeurs de blinis et de samovars ", observait Marina Tsvetaeva, toujours amère (Tsvetaeva, 2003).

En fait, la contemplation morose et idéalisée d'un passé révolu conduisait à une impasse. Henri Troyat, conscient de ce danger, disait de ses confrères : "Partis de Russie, ils n'étaient arrivés nulle part, ils continuaient à vivre dans une zone intermédiaire et abstraite. C'était l'enfer glacé des apatrides " (Troyat, 1976). Khodassevitch se sentait impuissant : "Ici, je ne suis capable ni de vivre ni d'écrire, et là-bas, je n'en ai plus le droit "(Berberova, 1992). Certains qui tenaient encore la plume craignaient que leur mémoire ne s'effilochât ou ne se transformât en un jeu de stéréotypes artificiels. Trofimoff voyait nombre d'écrivains comme des drogués, esclaves d'un rêve nocif :

" Je connais le danger de ces contemplations rétrospectives, elles nous ensevelissent dans un monde factice, nous rangent parmi les fossiles; on finit par ne plus pouvoir vivre sans ces obsédants mirages comme d'autres ne sauraient se passer de morphine " (Trofimoff, 1937).

La dépendance à l'égard d'un pays trop aimé et perdu amenait souvent au désespoir, "notre halte dernière ", selon I'expression de Georges Ivanov (Etkind, 1988). Alexandre Kouprine se lamentait : "La vie d'émigré $m^{\prime}$ 'a complètement annihilé et l'éloignement de ma patrie $m^{\prime}$ 'a écrasé l'âme contre le sol [...]. Je ne 
peux rien faire sans la Russie (Etkind, 1988). Pour ne pas sombrer définitivement, la plupart des écrivains mûrs ou âgés établissaient des comparaisons fréquentes entre leur pays d'origine et la France, toujours au détriment de cette dernière. Ils reprochaient à la patrie des droits de I'homme d'avoir failli à sa réputation, de n'avoir pas accordé aux exilés les droits et même les honneurs qu'ils méritaient. Dimitri Sesemann observait : "La médiocrité matérielle de notre existence suscitait un vif ressentiment à l'égard d'une société que nous jugions indigne de sa réputation, voire de sa vocation " (Sesemann, 2001). Marina Tsvetaeva critiquait même les forêts françaises, car on y rencontrait toujours des promeneurs qui l'empêchaient de s'enfermer dans l'altière solitude qu'elle trouvait dans immensités boisées de son pays. Banine décrivait les partis-pris antifrançais de son ami Ivan Bounine:

" Je lui reprochais son allergie à tout ce qui n'était pas russe (...). II dépérissait d'ennui avec les étrangers. Le seul fait d'user d'une langue autre que la sienne lui était un supplice ; d'ailleurs ce n'était pas l'effet du hasard qu'il parlât mal le français, mais une autre manière de marquer son aversion (Banine, 1968).

Pour surmonter la nostalgie et le désespoir, les écrivains ne se contentaient pas de dénigrer la France, car ils sentaient plus ou moins nettement qu'ils devaient trouver des raisons de vivre et d'espérer. Plusieurs pensèrent qu'il fallait dépasser le regret stérile du temps passé, oublier les aspects secondaires de la vie ancienne pour comprendre l'essence profonde de la patrie, cerner les contours de la Russie idéale et éternelle. Les épreuves de l'exil, la cruelle et brutale séparation d'avec la terre natale semblaient offrir une voie d'accès à l'essentiel. Dimitri Merejkovski expliqua comment s'opérait cette découverte :

"Ce n'est qu'à l'étranger que nous avons appris ce que signifie la Russie. Nous avons perdu la vue extérieure, mais nous avons ouvert les yeux intérieurs, et nous avons vu la

Russie invisible, la Terre sainte, promise [...]. II faut être privé de sa terre pour l'aimer d'un amour qui n'est pas terrestre. Notre amour infini, non terrestre, de la Russie, voilà ce qui fait notre force infinie " (Struve, 1996).

Plusieurs clefs se présentaient pour accéder à cette sorte de Russie archétypale. L'une des plus évidentes consistait à retracer la vie des grands hommes du passé qui avaient, chacun à sa manière, incarné l'âme nationale. C'est pourquoi parurent de nombreuses biographies consacrées notamment à Pouchkine, Dostoïevski, Gogol, Tourgueniev, les grands saints orthodoxes. Les études historiques permettaient également une approche de l'essentiel. À ce titre, les écrivains se consacrèrent particulièrement à une analyse des révolutions, celle de 1917 surtout, pour comprendre le rôle de l'individu dans les grandes convulsions du passé. Ces ouvrages visaient à montrer que I'homme possède une influence limitée sur le cours des choses et qu'il n'existe pas de déterminisme historique. Ainsi l'intelligentsia était excusée pour n'avoir pas prévu ou empêché l'effondrement de la Russie tant aimée. Au sein de la production littéraire de l'exil, de nombreuses autobiographies cristallisèrent la mémoire et, sous couleur de témoignage, s'attachèrent à définir la vraie Russie, celle d'avant 1917. La description, également autobiographique, de la vie menée en France, établissait un parallèle avec la patrie perdue, peinte sous un jour de plus en plus fantasmatique à mesure que le temps passait, cela pour faire ressortir les traits fondamentaux de la Russie. Dans l'évocation du passé, les écrivains glissaient 
de nombreux éléments de leur vie personnelle, ainsi firent Alexandre Kouprine, Ivan Chmeliov (Le Soleil des morts, 1923 ; Pèlerinage, 1931), Alexis Remizov (Sur champ d'azur, 1927), Ivan Bounine dans un de ses livres les plus marquants, La Vie d'Arseniev (1938), Boris Zaïtsev dans sa tétralogie Le Voyage de Gleb (19371953) dont il disait que le vrai personnage était la Russie éternelle.

\section{La jeune génération et l'ouverture à l'occident}

Contrairement à leurs aînés, les jeunes écrivains montraient de l'intérêt pour I'occident et la France. Cette attitude d'ouverture tenait d'abord au fait que ces jeunes, ayant vécu moins longtemps en Russie, étaient moins marqués par l'influence du pays d'origine. Ils n'avaient à regretter ni une influence intellectuelle qu'ils n'avaient pas encore conquise ni un public qui les connaissait à peine. Beaucoup pensaient que l'occident pouvait peut-être leur dispenser un enseignement et que celui-ci, ajouté à la tradition russe, donnerait naissance à de nouvelles formes littéraires. Aussi se mirent-ils à lire attentivement les œuvres de Joyce, récemment traduites en français, de Kafka et, chez les auteurs français, celles de Gide, Céline, Mauriac et surtout Proust. Les jeunes se montraient très sensibles à l'exceptionnelle réputation de la France et au rayonnement culturel de celle-ci. Pour le danseur Serge Lifar, Paris était "la capitale du monde " (Lifar, 1935). En s'installant dans cette ville, tous réalisaient une ardente aspiration. Ainsi, Banine, venue du lointain Azerbaïdjan, confiait que, pour apprécier à son prix un séjour dans la capitale de la France, il fallait avoir " rêvé de Paris pendant de longues, traînantes années, comme j'en avais rêvé au fond de ma ville natale où - sans faire de paradoxe - j'ai vécu mon vrai exil " (Banine, 1990). Katia Granoff assurait que " la France vous reçoit, vous nourrit de sa culture et vous fait citoyen à part entière " (Granoff, 1976). Les jeunes célébraient tout particulièrement la liberté dont ils jouissaient en France. La plupart en effet se plaignaient d'avoir souffert de l'autoritarisme du régime tsariste et de l'Église orthodoxe, puis de la dictature soviétique. Ils jouissaient de pouvoir désormais parler de tout et de ne subir aucune entrave à leur création artistique. Lifar concluait: "J'étais un libre citoyen de l'univers dans la plus libre des capitales " (Lifar, 1935).

Cependant les jeunes n'entendaient pas renier leur héritage culturel ; ils pensaient même que la confrontation de leurs traditions avec celles de l'occident permettrait de mieux comprendre la singularité et la valeur du legs russe. Ils voulaient d'autant plus rester fidèles à celui-ci qu'ils s'en sentaient, autant que les aînés, dépositaires. Cet état d'esprit se renforça dans les années 1930, car les jeunes se convainquirent alors que le régime soviétique se fortifiait et détruisait les valeurs de la vieille Russie. Le retour devenant de plus en plus aléatoire, les exilés se sentaient tenus par le devoir de maintenir en vie les traditions authentiques de leur patrie.

Souhaitant rester fidèles à la Russie tout en recevant ce que la France pouvait leur donner, les jeunes exilés se construisaient généralement dans le moule d'une identité complexe, partagée entre deux pôles. Henri Troyat en prit conscience très tôt, dès ses années de lycée : 
"Mes camarades ne changeaient pas de pays quand ils sortaient du lycée pour rentrer chez eux. Moi, en regagnant la maison, je quittais la France pour me plonger en Russie. Je vivais la moitié du jour à Paris et la moitié du jour à Moscou, un pied sur la terre ferme française, l'autre sur les nuages russes " (Troyat, 1976).

Quant à savoir si l'une des deux influences l'emportait, la plupart hésitaient à répondre. Marina Tsvetaeva confiait : " Ici, je suis inutile, là-bas, je suis impossible "(Desanti, 1994). Irène Nemiroski, après vingt ans de séjour en France, avouait à un journaliste : " Je désire, j'espère, je crois être un écrivain plus français que russe [...]. II m'est impossible de distinguer où finit I'un, où commence l'autre "(Les Nouvelles littéraires, 30 mars 1940). Dans cette ambiguïté identitaire, le choix de la langue apparaissait très révélateur. Le russe, symbole de la patrie perdue, était quasiment sacralisé et pieusement transmis aux enfants. Le résultat de révélait variable selon les individus. Ceux qui apprenaient le français après leur arrivée dans la terre d'exil se montraient parfois peu réceptifs ou découvraient un nouvel idiome qu'ils jugeaient difficile, surtout quand ils entreprenaient la rédaction d'un livre. Aux apprentis poètes qui maîtrisaient plus ou moins la langue de Molière, Nina Gourfinkel disait : "Vous ferez peut-être des poèmes, jamais de la poésie " (Gourfinkel, 1953). En revanche, ceux qui étaient déjà francophones avant de prendre le chemin de l'exil se sentaient parfaitement à l'aise ; tel était le cas de Vladimir Nabokov, élevé par une gouvernante suisse francophone, Joseph Kessel, Romain Gary, toujours premier en français au lycée, HenriTroyat, grand lecteur de la comtesse de Ségur dans sa première enfance. Aussi ce dernier pourra-t-il dire : "Je n'ai pas choisi le français. Il s'est imposé à moi " (Troyat, 1976). Katia Granoff affirmait : "Je suis poète français " (Granoff, 1976). L'évolution vers la langue de la nouvelle patrie se révélait d'autant plus naturelle que les exilés étaient coupés des sources vives de leur parler natal et immergés dans un univers totalement francophone. Zoé Oldenbourg fut profondément marquée par les vers de Racine que, pourtant, son entourage familial n'admirait pas particulièrement : "Cela chantait. Cela vous entrait dans la tête sans effort. Je connaissais par cœur tout le rôle de Phèdre, les trois quarts de la tragédie d'Andromaque, des scènes de Britannicus, de Bajazet, des fragments d'Iphigénie et de Mithridate "(Oldenbourg, 1982). Bientôt, elle se rendit compte qu'elle pensait dans la langue de Racine : "En écrivant en russe j'avais la sensation de traduire du français " (Oldenbourg, 1977). Nabokov, aussi à I'aise en russe qu'en français et en anglais tirait cette conclusion : "Je suis convaincu qu'il arrive un moment dans la vie d'un artiste où il cesse d'avoir besoin de sa patrie " (Raguet-Bouvard, 2000).

Les œuvres produites par les jeunes écrivains russes furent marquées par les contacts qu'ils avaient noués avec la culture française. Ils décrivirent tout naturellement leur expérience de l'exil sous forme de journaux ou de souvenirs, parfois romancés. Parmi les publications les plus marquantes peuvent être citées, entre autres, Nuits de princes, brillant roman à clefs relatant les expériences de l'auteur, C'est moi qui souligne, autobiographie de Nina Berberova et, du même écrivain, Les Chroniques de Billancourt où était relatée la vie des ouvriers russes travaillant aux usines Renault, La Promesse de l'aube, récit plus ou moins fidèle de la jeunesse de Romain Gary. Ces ouvrages brossaient le tableau du retournement de fortune dont les exilés souffraient, de leurs expériences identitaires, de l'évolution de leur mentalité et de leurs comportements. Dans beaucoup de ces peintures, les jeunes se présentaient comme des victimes 
solitaires, des prophètes prêchant dans le désert, raillés, voire détestés, par leurs aînés murés dans leur égoïsme ou par les Français indifférents. En vérité ce mythe du poète maudit possédait deux origines. II était à la fois présent dans la littérature russe traditionnelle qui évoquait la figure du maître à penser incompris, parfois persécuté, et dans les œuvres romantiques françaises du $\mathrm{XIXe} \mathrm{siècle.} \mathrm{Ainsi} \mathrm{les} \mathrm{jeunes} \mathrm{conceptualisaient} \mathrm{l'amère} \mathrm{expérience} \mathrm{de} \mathrm{l'exil} \mathrm{selon}$ deux grilles d'analyse, celle qu'ils avaient amenée avec eux et celle qu'ils avaient trouvée en France. Leur appréhension pessimiste du temps présent fut encore aggravée par le sombre bilan dressé par Paul Valéry au lendemain des horreurs vécues durant la Grande Guerre : dans La Crise de l'esprit (1919), I'auteur soulignait la faillite des valeurs traditionnelles et du rationalisme.

Des signes d'ouverture à l'occident par un dépassement des traditions furent aussi enregistrés dans la création poétique. Dans ce domaine, le principal représentant de la vieille génération, Vladislav Khodassevitch, adepte du néo-classicisme, demandait la perfection formelle se traduisant notamment dans une versification raffinée. Mais les jeunes, comme Georges Adamovitch, Georges Ivanov, Boris Poplavski, "le Rimbaud de l'émigration ", influencés par les recherches modernes qu'ils découvraient en France, refusaient la voie suggérée par le vieux maître. S'exprimant surtout dans la revue Tchiska, ils refusaient les débats jugés passéistes sur la forme et voulaient réfléchir à ce qui leur paraissait essentiel, le but de la vie, le tragique de la condition humaine, le monde dans lequel ils vivaient et non la Russie défunte. Pour atteindre ces objectifs, ils répudiaient les contraintes formelles qui bridaient les épanchements personnels ; ils affirmaient que, comme le montraient les versificateurs contemporains travaillant en France, la poésie authentique devait s'affranchir du carcan des contraintes.

Un petit groupe d'intellectuels russes trentenaires, les eurasiens, refusaient cependant de se mettre à l'école de l'occident. Ces jeunes, surtout le prince Nicolas Troubetskoï et le prince Dimitri Sviatopolk-Mirski, rappelaient que la Russie se trouve à cheval sur l'Europe et l'Asie. Selon eux, c'étaient les tribus mongoles venues de l'Est qui avaient construit l'identité du vieil empire. Mais Pierre le Grand avait européanisé la Russie, ainsi coupée de ses racines. La Providence avait peut-être utilisé le bras bolchevik pour abattre la Russie pétrinienne et la ramener à son moule originel. Moscou exploita cette ouverture qu'offrait le mouvement eurasien, le pénétra et réussit convaincre certains membres du groupe de se mettre à son service. Cette manœuvre entraîna un schisme en 1931 ; certains Eurasiens gagnèrent I'URSS où ils furent en partie exécutés par les hommes de Staline, les autres, prudemment restés en France, reprirent leur vie d'écrivains plus ou moins ouverts à des expériences nouvelles. 


\section{Conclusion}

Les écrivains russes blancs connurent la difficile condition de l'exil, une fréquente gêne matérielle, la nostalgie de la patrie perdue, le désespoir parfois. Le pessimisme se révélait particulièrement fort, car ces intellectuels avaient bien conscience de n'avoir aucune prise sur leur destin. Ils se savaient proscrits de leur pays, sans grande chance d'y revenir, sauf à courir de graves périls, contrairement à des travailleurs émigrés pouvant en principe circuler librement. Ils souffraient aussi de ce que, malgré leur témoignage, une partie de l'intelligentsia française montrait de l'intérêt, parfois même de la sympathie, pour l'expérience soviétique.

Les exilés parvinrent cependant à nouer des contacts dans le pays d'accueil. Ils bénéficièrent de la mode russe des années 1920 et de la solidarité de nombreux écrivains français parmi les plus connus. Ils n'en éprouvèrent pas moins un grand sentiment de solitude. Les plus âgés, comme Bounine, se repliaient volontairement et refusaient toute forme d'intégration. Certains jeunes, comme les Eurasiens, rejetaient les valeurs occidentales. D'autres, soumis à l'influence du modèle traditionnel russe et à celle des écrivains français, construisaient l'image de l'intellectuel solitaire et maudit.

Plus fondamentalement, les exilés se trouvaient soumis à une redoutable épreuve identitaire. Certes, comme beaucoup d'émigrés, ils étaient écartelés entre deux cultures, celle du pays d'origine et celle de la France, ce que révélaient bien les choix linguistiques de chacun. Mais, en plus, ils restaient dans l'incapacité de retrouver leur public naturel et les sources vives de leur inspiration. Obligés de demeurer en France, ils faisaient retour sur eux-mêmes, s'interrogeaient sur l'identité russe, la possibilité d'être Russe hors de Russie, de créer en russe ou en qualité de Russe loin du pays natal.

Ces difficultés, matérielles et psychologiques, les divisions idéologiques fragmentant la communauté russe, le défaut d'intégration sociale et souvent culturelle n'entravèrent pas la créativité des écrivains exilés. Parmi leurs romans, leurs poésies, leurs mémoires figurent des chefs-d'œuvre. Ce n'est pas trahir la réalité que de reconnaître la qualité de ces œuvres, supérieures en moyenne à beaucoup d'écrits inspirés par le réalisme socialiste imposé en URSS. Pour les écrivains blancs, continuer à être eux-mêmes constituait une sorte de défi et une raison de survie. Marqués par l'amère épreuve de l'exil forcé, ils mobilisèrent toutes les ressources de leur mémoire. Coupés de leur patrie par le fossé politique, la distance et l'écoulement des années, ils se dépouillèrent et allèrent à l'essentiel. Ils ne s'enfermèrent pas seulement dans le culte du passé ; les plus jeunes surent renouveler les thèmes et les formes, sans renier leur identité. Cette littérature aux contours souvent épurés, à la fois riche de témoignages, d'émotion et d'esprit, garda les couleurs de la vie. 


\section{Références bibliographiques}

Banine (1990) Jours parisiens, Montpellier, Gris banal, 209 p.

Banine (1968) La France étrangère, Paris, Desclée de Brouwer, 301 p.

Berberova Nina (1992) C'est moi qui souligne, Paris, J'ai lu, 573 p.

Bounine Ivan (2003) Les Allées sombres, Paris, Le livre de poche, 377 p.

Bounine Ivan (1950) Mémoires, Paris, Calmann-Lévy, 181 p.

Desanti Dominique (1994) Le Roman de Marina Tsvetaeva, Paris, Belfond, 387 p.

Etkind Efim, Nivat Georges, Serman Ilya et Strada Vittorio (1988-1990) Histoire de la littérature russe, tomes V et VI, Paris, Fayard.

Gazdanov Gaïto (2015) Une soirée chez Claire, Paris, Viviane Hamy, 174 p.

Gazdanov Gaïto (1991) Chemins nocturnes, Paris, Viviane Hamy, 264 p.

Gourfinkel Nina (1953) Aux prises avec mon temps, Tome II. L'Autre patrie, Paris, le Seuil, 349 p.

Granoff Katia (1976) Mémoires, Paris, 10/18, 279 p.

Khodassevitch Vladislav (1991) Nécropole, Arles, Actes Sud, 247 p.

Krivochéine Nina (1987) Les Quatre tiers d'une vie, Paris, Albin Michel, 325 p.

Lifar Serge (1935) Du temps que j'avais faim, Paris, Stock, 244 p.

Livak Leonid (2002) Nina Berberova et la mythologie culturelle de l'émigration russe en France, Cahiers du monde russe, 43 (2-3), pp. 463-478.

Nabokov Vladimir (1999) Parti pris, Paris, Laffont, 299 p.

Nabokov Vladimir (1961) Autres rivages. Souvenirs, Paris, Gallimard, 343 p.

Oldenbourg Zoé (1982) Le Procès du rêve, Paris, Gallimard, 324 p.

Oldenbourg Zoé (1980) La Joie-souffrance, Paris, Gallimard, 768 p.

Oldenbourg Zoé (1977) Visages d'un autoportrait, Paris, Gallimard, 416 p.

Raguet-Bouvart Christine (2000) Vladimir Nabokov, Paris, Belin, 127 p.

Sarraute Nathalie (1983) Enfance, Paris, Gallimard, 276 p.

Schor Ralph (2013) Écrire en exil. Les écrivains étrangers en France, 1919-1939, Paris, CNRS-Editions, $352 \mathrm{p}$.

Schor Ralph (1985) L'Opinion française et les étrangers, 1919-1939, Paris, Publications de la Sorbonne, $762 \mathrm{p}$.

Sesemann Dimitri (2001) Les Confessions d'un métèque, Paris, Lattès, 280 p.

Stravinki Igor (1935) Chroniques de ma vie, Paris, Denoël, 240 p.

Struve Nicolas (1996) Soixante-dix ans d'émigration russe, 1919-1989, Paris, Fayard, $304 \mathrm{p}$. 
Tchernoff Louda (1938) Dans le creuset des civilisations. Des prodromes du bolchevisme à une Société des nations, Paris, Rieder, 360 p.

Trofimoff André (1937) Du musée impérial au marché aux puces, Paris, Les Argonautes, $213 \mathrm{p}$.

Troyat Henri (1976) Un si long chemin, Paris, Stock, 217 p.

Tsvetaeva Marina (2008) Les Carnets : 1913-1939, Paris, Éditions des Syrtes, $1126 \mathrm{p}$.

Tsvetaeva Marina (2004) Lettres du grenier de Wilno : lettres de Marina Tsvetaeva à Natalia Hajdukiewicz, Paris, Éditions des Syrtes, 139 p.

Tsvetaeva Marina (2003) Lettres à Anna, Paris, Éditions des Syrtes, 249 p.

Țsvetaeva Marina et Pasternak Boris (2005) Correspondance, 1922-1936, Paris, Éditions des Syrtes, 679 p.

Waegemans Emmanuel (2003) Histoire de la littérature russe, Toulouse, Presses universitaires du Midi, $450 \mathrm{p}$. 


\section{Ralph Schor}

\section{Les écrivains russes blancs en France. Un entre-deux identitaire (1919-1939)}

La révolution de 1917 lança sur les routes de l'exil de nombreux écrivains russes dont certains de première importance comme Bounine, premier Russe lauréat du prix Nobel de littérature (1933), Nabokov, Kouprine, Merejkovski, Troyat, Zoé Oldenbourg, MarinaTsvetaeva, Nina Berberova, Irène Némirosvki, etc. Les exilés bénéficiaient de facteurs favorables : la mode russe qui se développa en France dans les années 1920, la présence de réseaux intellectuels de communication, des rencontres avec les Français. Mais la mode se dissipa dans les années 1930, I'intérêt pour I'URSS grandit, des divisions affaiblirent la communauté russe, la solitude et la gêne matérielle furent durement ressenties. La vieille génération éprouva particulièrement la douleur de l'exil. Beaucoup s'interrogeaient sur I'avenir d'une littérature d'exil. La nostalgie du passé et le désespoir semblaient conduire à une impasse. Un grand nombre d'écrivains se réfugièrent dans une Russie idéale et éternelle, ce qui conduisait souvent au dénigrement de la France. Cet état d'esprit inspira beaucoup d'autobiographies qui cristallisaient la mémoire, d'études et de romans historiques. La jeune génération qui avait vécu moins longtemps en Russie marqua un intérêt plus grand pour l'occident, s'ouvrit davantage à la culture française et apprécia la liberté qu'offrait la vie en France. Cependant beaucoup gardèrent une identité double, à la fois russe et française. Sur le plan de la création littéraire, mis à part les Eurasiens hostiles à l'occident, l'inspiration des jeunes se renouvela et s'orienta notamment vers l'analyse de l'existence menée par les émigrés en France. Un débat sur la poésie opposa ces jeunes à leur aîné, Khodassevitch, plus traditionaliste.

\section{White Russian Writers in France. A Half-Way Identity (1919-1939)}

The 1917 revolution throws to exile roads many Russian writers as Bounine, first Russian Nobel of literature (1933), Nabokov, Kouprine, Merejkovski, Troyat, Zoe Oldenbourg, Marina Tsvetaeva, Nina Berberova, Irene Nemirovski, etc. Exiles have favourable factors: Russian fashion during the 1920's, intellectual networks of communication, meetings with French people. But fashion disappears during the 1930's, interest for URSS increases, divisions weaken Russians, solitude and poverty are important. Old generation is particularly unhappy. Exile literature is it possible? Many take refuge in ideal Russia and denigrate France. So writers publish autobiographies and studies and historical novels. Young generation who knows less Russia is must interested by occident, French culture and liberty in this land. But many have double identity, Russian and French, except Eurasians. Inspiration of young writers is analysis of their French experience and poetic discussions. 


\section{Escritores rusos blancos en Francia. Una identidad intermedia (1919-1939)}

La revolución de 1917 arroja al exilio a muchos escritores rusos, algunos de gran importancia como Bunin, el primer ganador ruso del Premio Nobel de Literatura (1933), Nabokov, Kuprin, Merezhkovsky, Troyat, Zoe Oldenburg, Marina Tsvetaeva, Nina Berberova e Irene Nemirosvki entre otros. Estos exiliados se beneficiaron de factores favorables: la moda rusa que se desarrolló en Francia en la década de 1920, la presencia de redes intelectuales de comunicación, los encuentros con los franceses. Pero la moda desapareció en la década de 1930, el interés por la URSS aumentó, las, divisiones debilitaron la comunidad rusa, la soledad y la pobreza se sintieron profundamente. En particular, la vieja generación experimentó el dolor del exilio. Muchos cuestionaron el futuro de una literatura del exilio. La nostalgia por el pasado y la desesperación parecían llevar a un callejón sin salida. Muchos escritores se refugiaron en una Rusia ideal y eterna, que a menudo conducía a la denigración de Francia. Este estado de ánimo inspiró muchas autobiografías que cristalizaron la memoria, los estudios y las novelas históricas. La generación más joven que había vivido menos tiempo en Rusia mostró un mayor interés en Occidente, más abierto a la cultura francesa y a la libertad que ofrece la vida en Francia. Sin embargo, muchos conservaron una doble identidad, tanto rusa como francesa. En cuanto a la creación literaria, con independencia de los euroasiáticos hostiles a Occidente, la inspiración de los autores jóvenes se renovó y se dirigió especialmente al análisis de la existencia de los emigrantes en Francia. Un debate sobre la poesía opuso a estos jóvenes a su antecesor, Khodassevich, más tradicionalista. 